\title{
Renal Kallikrein Activation and Renoprotection after Dual Blockade of Renin-Angiotensin System in Diet-Induced Diabetic Nephropathy
}

\author{
Xia Zou, ${ }^{1}$ Xiao-xi Zhang, ${ }^{1}$ Xin-yu Liu, ${ }^{1}$ Rong Li, ${ }^{1,2}$ Min Wang, ${ }^{1}$ Wei-jie Wu, \\ Yi Sui, ${ }^{3}$ and Hai-lu Zhao ${ }^{1}$ \\ ${ }^{1}$ Center for Diabetic Systems Medicine, Guangxi Key Laboratory of Excellence, Guilin Medical University, Guilin 541004, China \\ ${ }^{2}$ Molecular Endocrinology and Toxicology Laboratory, Department of Biology, Hong Kong Baptist University, \\ Kowloon 999077, Hong Kong \\ ${ }^{3}$ Department of Endocrinology, Guangdong Hospital of Traditional Chinese Medicine, Guangzhou 510120, China
}

Correspondence should be addressed to Hai-lu Zhao; zhaohailu9@126.com

Received 22 October 2014; Revised 19 January 2015; Accepted 9 March 2015

Academic Editor: Konstantinos Kantartzis

Copyright (c) 2015 Xia Zou et al. This is an open access article distributed under the Creative Commons Attribution License, which permits unrestricted use, distribution, and reproduction in any medium, provided the original work is properly cited.

\begin{abstract}
Purpose. The objective of this study is to investigate the effect of dual blockage of renin-angiotensin system (RAS) on renal kallikrein expression and inflammatory response in diabetic nephropathy (DN). Methods. Rats were randomly divided into 5 groups with 10 rats in each group: normal control; DN model induced by high fat and high sucrose diets; and DN treated with either benazepril $10 \mathrm{mg} / \mathrm{kg} / \mathrm{d}$, irbesartan $30 \mathrm{mg} / \mathrm{kg} / \mathrm{d}$, or both. After 8-week treatment, we examined changes in the kidney histopathology, function and immunohistochemical stain of kallikrein, macrophage marker CD68, and profibrotic markers transforming growth factor(TGF-) $\beta$ and $\alpha$-smooth muscle action (SMA). Results. DN rats showed enlarged kidneys with glomerulosclerosis, interstitial chronic inflammation and fibrosis, and proteinuria. All the pathological damage and functional impairments were improved after the RAS blockades (all $P<0.05$ ). Compared with monotherapy, combined treatment further alleviated the kidney impairments in parallel to increased tubular immunoreactivity for kallikrein and decreased immunopositive cells for CD68, TGF- $\beta$, and $\alpha$-SMA. Conclusion. The renoprotective effects of the dual RAS blockade in diabetic nephropathy may be attributed to improved tubular kallikrein expression and interstitial inflammatory response.
\end{abstract}

\section{Introduction}

Diabetic nephropathy (DN) is one of the most common microvascular complications of diabetes mellitus (DM) and a leading cause of end-stage renal disease (ESRD) [1]. China currently has approximately 100 million adults with type2 diabetes and 150 million people with prediabetes [2, 3]. Alarmingly, 120 million Chinese adults may suffer from chronic kidney disease manifested predominantly as albuminuria [4]. Albuminuria is the major determinant of DN and an important risk factor for cardiovascular disease in patients with diabetes. Indeed, hospitalized Chinese patients with type- 2 diabetes showed $44 \%$ of microalbuminuria, $12 \%$ of macroalbuminuria, and $17 \%$ of cardiovascular disease [5]. Therefore, type-2 diabetes and chronic kidney disease have become major public health problems in China.
These chronic noncommunicable diseases are mutually interrelated with unmet medical needs [6].

Renin-angiotensin system (RAS) plays a key role in the pathogenesis of diabetic nephropathy. RAS blockage including angiotensin-converting enzyme inhibitors (ACEI) and angiotensin receptor blockers (ARB) is currently used for DN treatment $[7,8]$. Furthermore, $D N$ prevention also relies substantially on the prescription of ACEI and ARB in clinical settings [9]. ACEI such as benazepril have beneficial effects on DN independent of blood pressure-lowering effect and plasma angiotensin II levels [10]. Similarly, ARB such as irbesartan also reveal renoprotective effects in landmark clinical trials [11-14]. In this regard, researches of dual blockade with combined ACEI and ARB are ongoing to maximize the beneficial effects and minimize the adverse events. 
The disruption of the RAS pathway leads to negative feedback and compensatory kallikrein system activation [1517]. Abnormal activation of kallikrein excitation peptide system (KKS) mainly induces DN progression [10]. Studies have found that heart tissue kallikrein activity significantly increases the effect of ACEI in type-2 diabetes [18], indicating mechanistic interaction between KKS and RAS [19].

The aim of the present study was to investigate the effect of combined intervention with ACEI-benazepril and ARBirbesartan on experimental DN rats. We hypothesized that the combined therapy could protect against hyperglycemiainduced chronic renal impairments through renal tissue kallikrein system activation and inflammatory pathway inhibition.

\section{Materials and Methods}

2.1. Animals and Diets. Male Sprague Dawley rats $(230 \pm 20 \mathrm{~g}$ body weight) were purchased from Guilin Medical Laboratory Animal Center. The animals were housed in a specific pathogen-free (SPF) laminar flow cabinet. Temperature and humidity were maintained at $25 \pm 1^{\circ} \mathrm{C}$ and $50 \pm 5.0 \%$, respectively. The rats, which were exposed to light, were regulated in $12: 12 \mathrm{~h}$ light-dark cycle. The diet and sterilized water were used to feed these rats.

Ethical approval for animal studies was according to the Animal Experimentation Ethics Committee of Guilin Medical University. In this study, we used high fat and high sucrose diets (HFD) and conventional diet (5001 Rodent Diet; LabDiet, St. Louis, MO, USA). HFD contains 10\% lard, $20 \%$ sucrose, $2 \%$ egg yolk powder, $3 \%$ cholesterol, and $65 \%$ conventional diet.

2.2. Diet-Induced Diabetic Nephropathy and RAS Blockade. After adaptive feeding for one week, the rats were fed with conventional diet $(n=10)$ or HFD $(n=50)$ for 4 weeks. Then, the HFD-fed rats received intraperitoneal injection with low dose $(35 \mathrm{mg} / \mathrm{kg}$ ) of streptozotocin (Sigma, St. Louis, MO, USA) to hasten diabetic status. Streptozotocin was freshly prepared by dissolving in sterile sodium citrate-trisodium citrate buffer $(0.1 \mathrm{mmol} / \mathrm{L}, \mathrm{PH} 4.2)$. Meanwhile, the rats fed with conventional diet also received intraperitoneal injection of the same volume of the buffer. Six weeks later, DN rats were defined by the following criteria: (1) blood glucose level $\geqslant 16.7 \mathrm{mmol} / \mathrm{L}$; (2) 1.5 -fold increase in urine volume compared to the baseline; and (3) urinary albumin excretion rate beyond $30 \mathrm{mg} / 24 \mathrm{~h}[20,21]$. A total of $40 \mathrm{HFD}$-fed rats met the criteria and eventually were included in this study.

Subsequently, the rats were divided into 5 groups and each group had 10 rats: (1) normal control; (2) DN model control; (3) $\mathrm{DN}$ rats treated with benazepril $10 \mathrm{mg} / \mathrm{kg} / \mathrm{d}$; (4) $\mathrm{DN}$ rats treated with irbesartan $30 \mathrm{mg} / \mathrm{kg} / \mathrm{d}$; and (5) DN rats treated with both benazepril $10 \mathrm{mg} / \mathrm{kg} / \mathrm{d}$ and irbesartan $30 \mathrm{mg} / \mathrm{kg} / \mathrm{d}$. Blood glucose level was determined via taking blood from tail vein once a week, using the Precision Xtra blood glucose monitoring system (Alameda, CA, USA).

At the end of 8 -week treatments, $24 \mathrm{~h}$ urine from each group was collected using metal metabolism cage to calculate total urine volume and measure albumin excretion rate.
Fasting serum insulin concentrations were measured using enzyme immunoassay and rat insulin ELISA kit (Mercodia, Uppsala, Sweden). HOMA-IR (homeostasis model assessment of insulin resistance) index was estimated with the formula HOMA-IR $=$ fasting serum insulin $(\mathrm{mU} / \mathrm{L}) \times$ fasting serum glucose $(\mathrm{mmol} / \mathrm{L}) / 22.5$ [22]. Kidney index here reflects kidney hypertrophy estimated by the kidney weight/body weight $(\mathrm{mg} / \mathrm{g})$.

2.3. Immunohistochemistry, Light, and Fluorescence Microscopy. All the kidney tissue samples were fixed in $10 \%$ buffered formalin and embedded in paraffin. Serial sections $(4 \mu \mathrm{m})$ were cut perpendicular to the longest axis of the kidney. Sections were stained by primary antibodies including kallikrein 1, the phagocytic immunomarker CD68 (macrophage) [23], the proinflammatory and profibrotic markers transforming growth factor- (TGF-) $\beta 1$, and $\alpha$-smooth muscle action (SMA) (Santa Cruz, CA, USA), as described in our prior reports [22-24]. Stained slides were counterstained with either DAB (brown) for light microscopy or antifading DAPI (blue) for immunofluorescence microscopy, followed by examination with a Zeiss A2 imaging microscope (Carl Zeiss, Hamburg, Germany). When checking the stained slides, the pathologist was blinded for animal grouping.

2.4. Statistical Analyses. All data were analyzed using the SPSS 19.0 software. Comparisons among the five groups were analyzed by one-way analysis of variance (ANOVA), followed by Tukey post hoc multiple comparison test. Two-tailed $P$ values less than 0.05 were considered statistically significant. All data were presented as mean \pm SD.

\section{Results}

3.1. Characterization of the Diet-Induced Diabetic Nephropathy. Before injecting streptozotocin, the average concentrations of blood glucose between normal control and HFDfed groups were not significantly different. However, the HFD stimulated a significant increase in body weight, fasting serum insulin, and HOMA-IR (Table 1).

Six weeks after injecting the low dose of streptozotocin, HFD-fed rats $(n=40)$ showed hyperglycemia with average blood glucose level over $16.7 \mathrm{mmol} / \mathrm{L}$, polyuria with 1.5 fold increased urine volume, and albuminuria with urinary albumin excretion rate higher than $30 \mathrm{mg} / 24 \mathrm{~h}$. All the biochemical outcomes indicate establishment of DN model by HFD plus low dose streptozotocin.

3.2. Renoprotection by RAS Blockade. To evaluate renal function changes after RAS blockade, $24 \mathrm{~h}$ urine protein, blood urea nitrogen, and serum creatinine were determined. Compared with the normal controls, the DN rats showed kidney dysfunction echoed by elevated serum levels of $24 \mathrm{~h}$ urine protein (Figure 1(a)) and blood urea nitrogen (Figure 1(b)) and serum creatinine (Figure $1(\mathrm{c}))$ (all $P<0.05$ ). The renal dysfunction significantly improved after the treatments with ACEI, ARB, or both (Figure 1). Notably, the dual RAS blockade almost normalized the renal functions. Similar 
TABLE 1: Comparison of body weight, fasting blood glucose, blood glucose at 2 hours in OGTT, fasting plasma insulin, and insulin resistance index.

\begin{tabular}{|c|c|c|c|c|c|}
\hline Group & Body weight (g) & $\begin{array}{c}\text { Fasting blood } \\
\text { glucose }(\mathrm{mmol} / \mathrm{L})\end{array}$ & $\begin{array}{c}\text { Blood glucose at } 2 \\
\text { hours in OGTT } \\
(\mathrm{mmol} / \mathrm{L})\end{array}$ & $\begin{array}{l}\text { Fasting plasma } \\
\text { insulin }(\mathrm{mU} / \mathrm{L})\end{array}$ & $\begin{array}{c}\text { Insulin resistance } \\
\text { index }\end{array}$ \\
\hline $\begin{array}{l}\mathrm{CN} \\
n=10\end{array}$ & $370.80 \pm 14.44$ & $5.62 \pm 0.79$ & $5.88 \pm 0.97$ & $15.98 \pm 1.58$ & $4.01 \pm 0.86$ \\
\hline $\begin{array}{l}\text { HFD } \\
n=40\end{array}$ & $397.67 \pm 30.65^{*}$ & $5.69 \pm 0.61$ & $6.13 \pm 0.65$ & $26.32 \pm 2.05^{*}$ & $6.67 \pm 0.98^{*}$ \\
\hline
\end{tabular}

$\mathrm{CN}$ : normal diet control group; HFD: high fat and high sucrose diets group and ${ }^{*} \mathrm{P}<0.05$ compared with control group; $n$ : the number of samples; OGTT: oral glucose tolerance test.

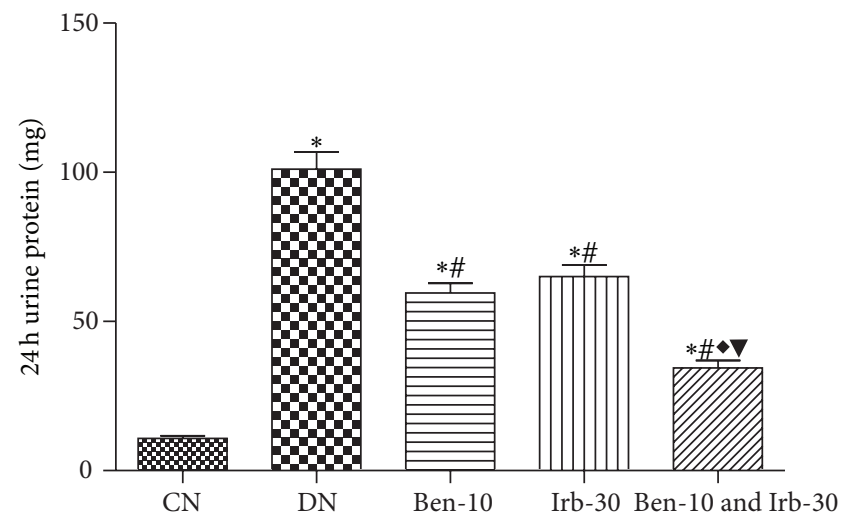

(a)

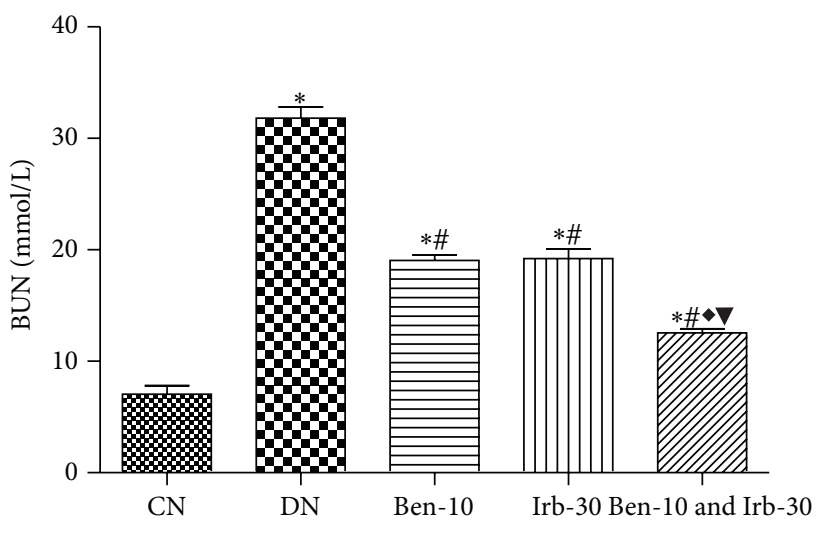

(b)

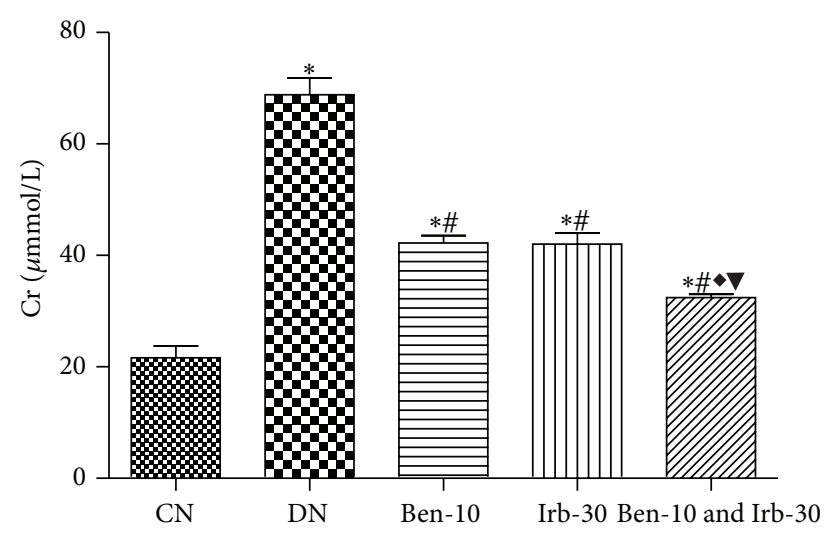

(c)

FIGURE 1: The effect of benazepril and irbesartan on the levels of $24 \mathrm{~h}$ urine protein (a), BUN (b), and Cr (c); CN: normal control group, group 1; DN: diabetic nephropathy control group, group 2; Ben-10, group 3 (benazepril $10 \mathrm{mg} / \mathrm{kg} / \mathrm{d}$ ); Irb-30, group 4 (irbesartan $30 \mathrm{mg} / \mathrm{kg} / \mathrm{d}$ ); and Ben-10 and Irb-30, group 5; and compared with group $1,{ }^{*} P<0.05$; compared with group $2,{ }^{\#} P<0.05$; compared with group 3, $P<0.05$; compared with group $4,{ }^{\nabla} P<0.05$; BUN: blood urea nitrogen; $\mathrm{Cr}$ : serum creatinine.

renoprotective effects were observed between the ACEI and ARB treatments (Figure 2(a)). Interestingly, obvious blood glucose lowering was achieved by the dual RAS blockade versus DN control and monotherapy.

Pathological changes in the DN rats included kidney hypertrophy reflected by the increased kidney index (Figure 2(b)), mild-to-moderate glomerulosclerosis, tubular atrophy with basement membrane thickening, arteriosclerosis, and interstitial fibrosis with chronic inflammatory infiltration (data not shown). Treatments with ACEI, ARB, or both generally ameliorated the pathological alterations. Consistent with the renal function improvement, pathological correction by the combined therapy was superior to any monotherapy.

3.3. Renal Immunoreactivity of Kallikrein. Figure 3 shows the immunoreactivity of kallikrein predominantly localized in renal tubular cells and generally negative in glomeruli. The DN control rats showed very fewer cells reactive for kallikrein (Figure 3). Tubular cells positive for kallikrein substantially 


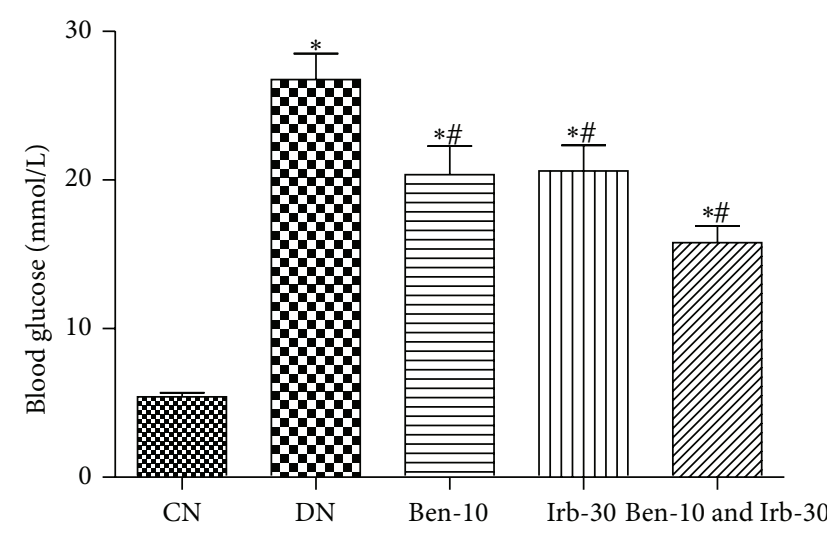

(a)

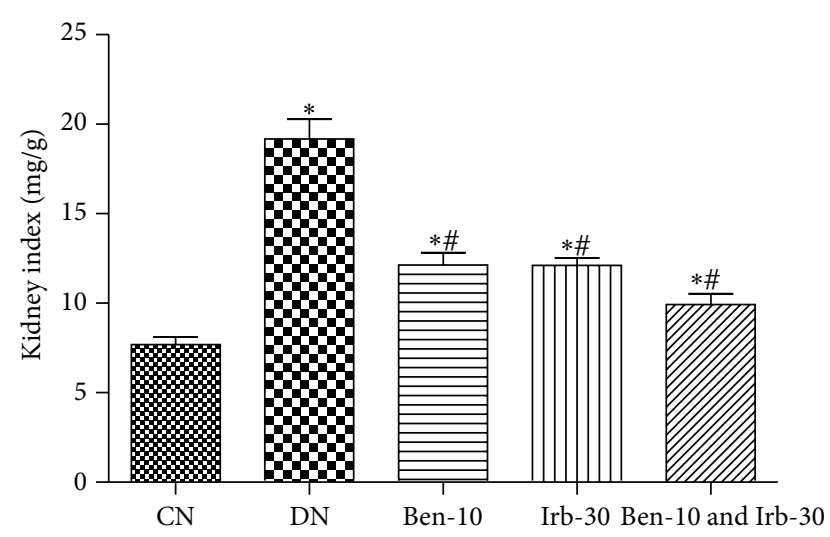

(b)

Figure 2: The effect of benazepril and irbesartan on the levels of blood glucose (a) and kidney index (b); CN: normal control group, group 1; DN: diabetic nephropathy control group, group 2; Ben-10, group 3 (benazepril $10 \mathrm{mg} / \mathrm{kg} / \mathrm{d}$ ); Irb-30, group 4 (irbesartan $30 \mathrm{mg} / \mathrm{kg} / \mathrm{d}$ ); and Ben-10 and Irb-30, group 5; and compared with group $1,{ }^{*} P<0.05$; compared with group $2,{ }^{*} P<0.05$.

TABLE 2: Expression of kallikrein 1 in renal tissue.

\begin{tabular}{lcc}
\hline Group & $\begin{array}{c}\text { The number } \\
\text { of samples }\end{array}$ & $\begin{array}{c}\text { Kallikrein } 1 \text { integral } \\
\text { optical density value }\end{array}$ \\
\hline 1 & 10 & $9.392 \pm 0.597$ \\
2 & 7 & $21.839 \pm 4.686^{*}$ \\
3 & 10 & $30.312 \pm 7.700^{* \#}$ \\
4 & 9 & $31.717 \pm 5.147^{* \#}$ \\
5 & 8 & $46.119 \pm 5.385^{* \# \bullet}$ \\
\hline
\end{tabular}

Group 1, normal control group; group 2, diabetic nephropathy control group; group 3, benazepril $(10 \mathrm{mg} / \mathrm{kg} / \mathrm{d})$; group 4 , irbesartan $(30 \mathrm{mg} / \mathrm{kg} / \mathrm{d})$; and group 5 , benazepril $(10 \mathrm{mg} / \mathrm{kg} / \mathrm{d}) \&$ irbesartan $(30 \mathrm{mg} / \mathrm{kg} / \mathrm{d}) ;$ and compared with group $1,{ }^{*} P<0.05$; compared with group $2,{ }^{\#} P<0.05$; compared with group $3,{ }^{\bullet} P<0.05$; compared with group $4,{ }^{\nabla} P<0.05$.

increased in rats which received the ACEI, ARB, or both (Table 2). The dual blockade was superior to monotherapy for stimulating kallikrein immunoreactivity.

3.4. Inflammatory CD68-Immunoreactive Cells. Cells immunoreactive for the inflammatory marker CD68 were mainly interstitial large macrophages intermixed with chronic inflammatory infiltrates (Figure 4). The CD68-positive cells were rare in normal control rats (Figure $4(\mathrm{a})$ ) but frequently seen as diffuse infiltrates in the DN rats (Figure 4(b)). Treatments with the RAS blockers prevented the chronic inflammatory infiltrates reactive for the phagocytic marker CD68. The dual blockade was better than the ACEI (Figure 4(c)) and ARB (Figure 4(d)) to diminish the CD68-stained cell infiltration (Figure 4(e)). In general, the density of CD68positive cells was in parallel to the severity of structural damage including glomerulosclerosis, arteriosclerosis, and interstitial fibrosis.

3.5. Inflammatory Transforming Growth Factor- $\beta$ and $\alpha$ Smooth Muscle Action. In general, chronic inflammatory cells immunoreactive for the profibrotic marker TGF- $\beta 1$ were interstitial macrophages frequently intermixed with infiltrating lymphocytes (Figure 5), consistent with the CD68 reactivity in Figure 4. In contrast, both interstitial fibroblasts and vascular smooth muscle cells were positive for the activated fibroblast marker $\alpha$-SMA. $\alpha$-SMA positivity localized mainly with arteriosclerosis and interstitial fibrosis whereas immunoreactivity of TGF- $\beta 1$ was accompanied by chronic inflammatory infiltration (Figure 5). Among the five groups of animals, the DN rats showed a few more cells positive for TGF- $\beta 1$ and $\alpha$-SMA than normal controls and rats given RAS blockers (Figure 5). Moreover, combined treatment appeared better than monotherapy in attenuating the immunofluorescence of TGF- $\beta 1$ and $\alpha$-SMA. Finally, the ACEI and ARB demonstrated similar effects on the immunofluorescence.

\section{Discussion}

This study shows tubular kallikrein activation and inflammatory amelioration likely underlying the renoprotective effects of RAS blockades in diet-induced diabetic nephropathy. Dual RAS blockade with both ACEI and ARB may delay the development of diet-induced diabetic nephropathy through compensatory kallikrein upregulation and inflammatory downregulation.

Both kallikrein-kinin system (KKS) and renin-angiotensin system (RAS) have been implicated in the pathogenesis of diabetic nephropathy (DN) $[25,26]$. The KKS serves as the physiological and pathophysiological counterbalance to the RAS. Tissue kallikrein 1 is a member of the tissue kallikrein family that is mainly responsible for the generation of kinins, while bradykinin (BK) is the principal kinin responsible for the physiological actions [26]. In the kidney, kallikrein 1 is confined to the tubules in both healthy and diabetic states. Consistent with our findings in this study, a reduction of renal kallikrein excretion has been found in diabetic individuals and patients with diabetic nephropathy [27]. Early clinical evidence has indicated a significantly lower urinary kallikrein excretion in type- 2 diabetic patients with nephropathy than 


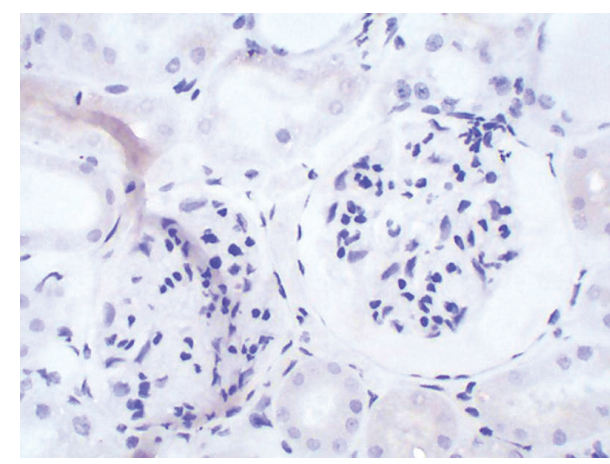

(a)

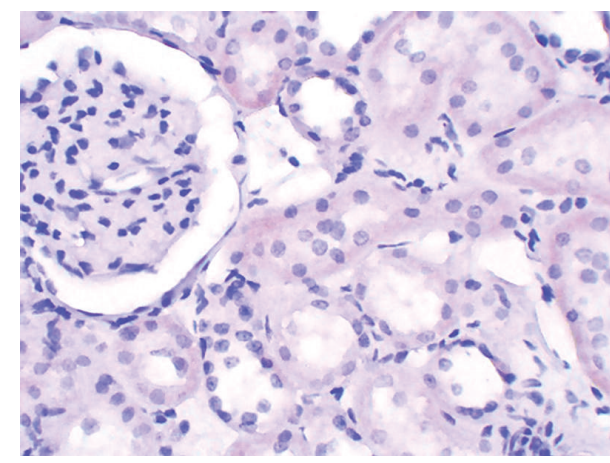

(c)

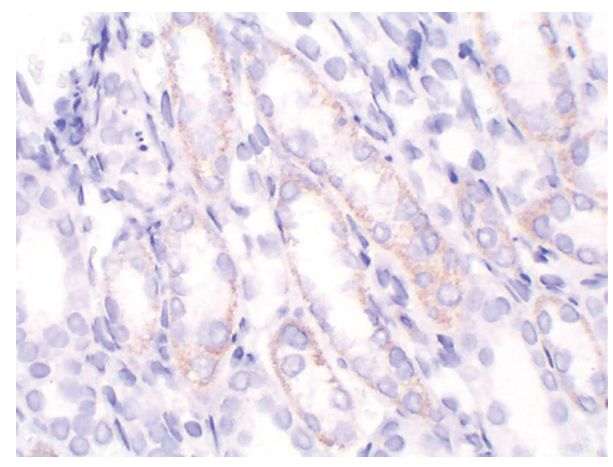

(b)

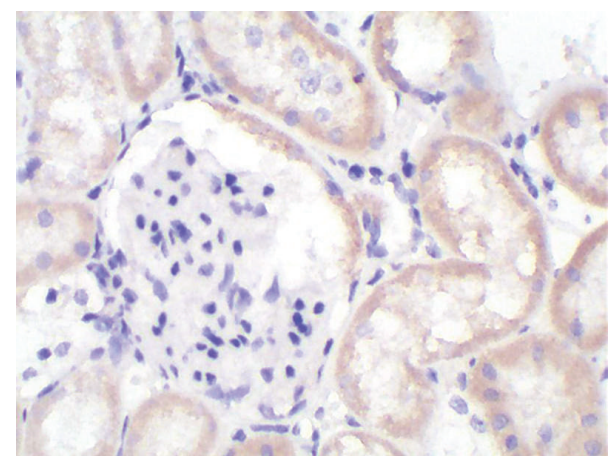

(d)

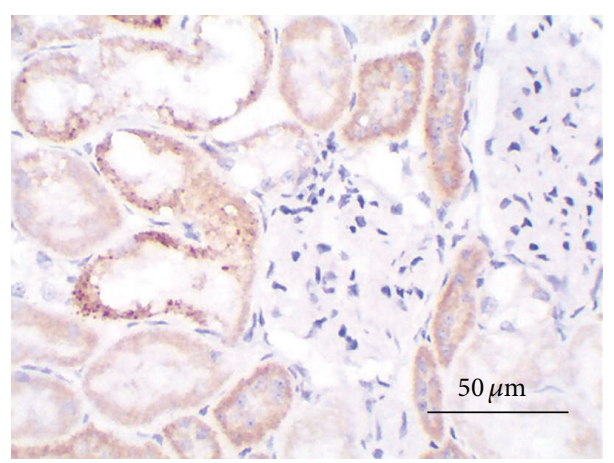

(e)

Figure 3: The immunohistochemical staining of kidney (HE 400x) in five groups. (a) Normal control group; (b) diabetic nephropathy control group; (c) benazepril (10 mg/kg/d); (d) irbesartan (30 mg/kg/d); and (e) benazepril (10 mg/kg/d) and irbesartan (30 mg/kg/d); scale bar, $50 \mu \mathrm{m}$.

in diabetic patients without nephropathy and in control subjects [28]. In experimental type-1 diabetes, kallikrein protects against the development of microalbuminuria [29]. In streptozotocin-induced diabetic rats, kidney tissue level and excretion of active kallikrein were reduced after 3 weeks compared with age-matched nondiabetic control rats, and despite increased kidney size, renal plasma flow was reduced in the diabetic rats [30]. Increased urinary BK levels found in severely hyperglycemic diabetic rats are related to increased filtration of components of the plasma KKS and renal kininogen synthesis in combination with decreased renal kinin-degrading activity [31]. All these findings are similar to the present study. Furthermore, activated KKS under diabetic condition may be beneficial in preventing podocyte loss in diabetic nephropathy [32]. Previous reports have shown that intravenous delivery of the human tissue kallikrein gene reduced blood pressure and plasma insulin levels in fructose-induced hypertensive rats with insulin resistance [33]. Furthermore, a recombinant adenoassociated viral vector expressing the human tissue kallikrein cDNA as a sole, long-term therapy could correct insulin resistance and prevent renal damage in streptozotocin-HFD-induced type2 diabetic rats [34]. In this study, the intensity of tubular kallikrein expression was related to the severity of diabetic nephropathy defined by polyuria, proteinuria, kidney hypertrophy, and chronic inflammatory infiltration. All these 


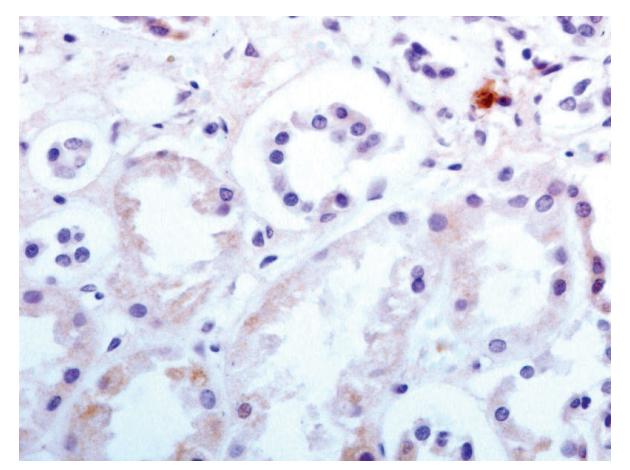

(a)

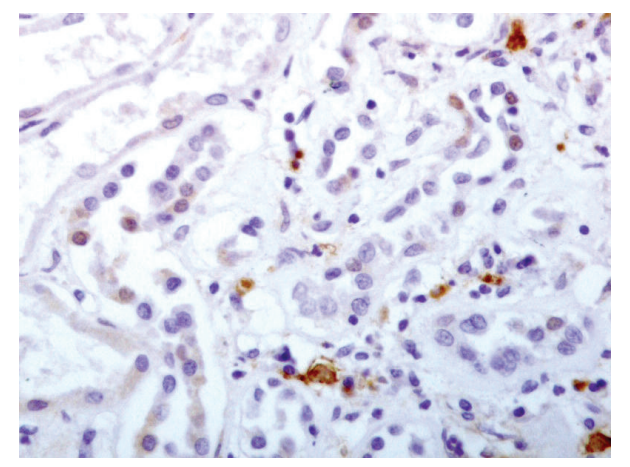

(c)

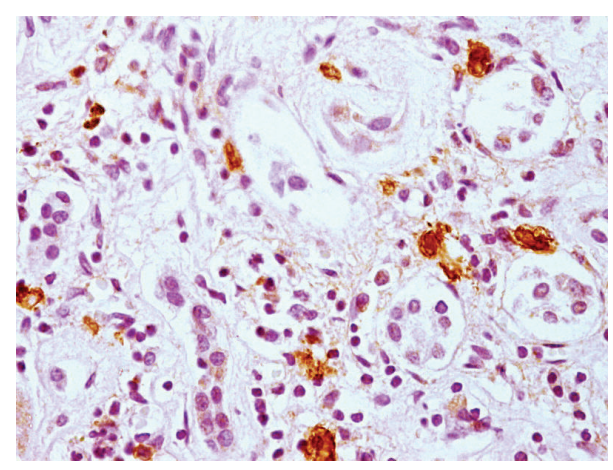

(b)

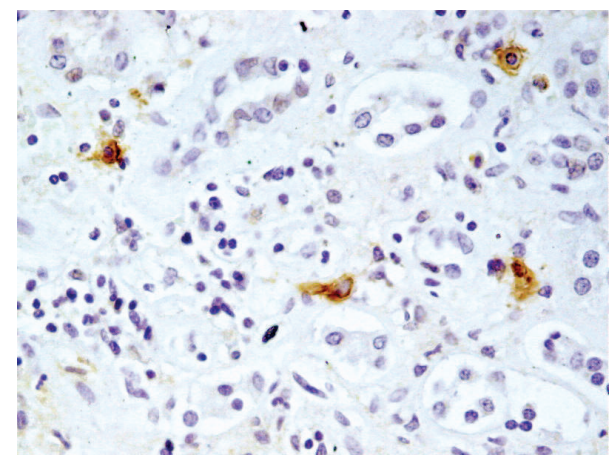

(d)

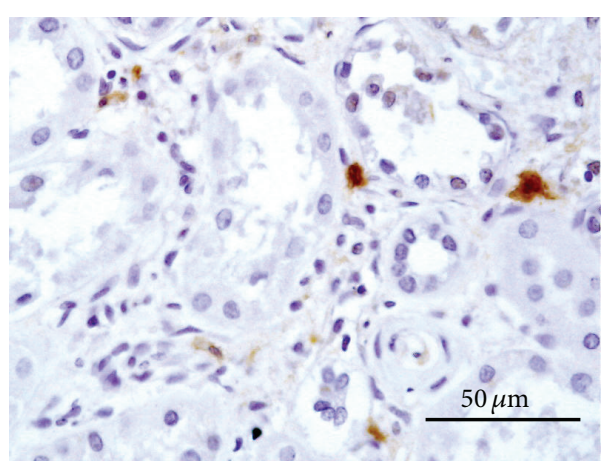

(e)

FIGURE 4: Light microscopy of immunohistochemical stain with CD68. Kidney tissue sections ( $4 \mu \mathrm{m})$ were stained with the phagocytic marker CD68 (brown) and examined under a Zeiss A2 imaging microscope (Carl Zeiss, Hamburg, Germany). Immunoreactive cells are mainly interstitial large macrophages intermixed with chronic inflammatory infiltrates. (a) Normal control (CN); (b) model of diabetic nephropathy that never had any treatment (DN); (c) treatment with benazepril $10 \mathrm{mg} / \mathrm{kg} / \mathrm{d}$ (Ben); (d) treatment with irbesartan $30 \mathrm{mg} / \mathrm{kg} / \mathrm{d}$ (Irb); (e) combined treatment with benazepril $10 \mathrm{mg} / \mathrm{kg} / \mathrm{d}$ and irbesartan $30 \mathrm{mg} / \mathrm{kg} / \mathrm{d}$ (Ben and Irb). Original magnification, $\times 200 ; \mathrm{scale}$ bar, $50 \mu \mathrm{m}$.

findings suggest that the KKS may serve as a therapeutic target in delaying the progression of diabetic nephropathy [35].

The present study also highlights the close interaction between the KKS and the RAS. Both urinary kallikrein excretion and kallikrein excretion rate $(24 \mathrm{~h}$ excretion of urinary kallikrein/24 h creatinine clearance) in hypertensive diabetic patients with nephropathy were significantly lower than in normotensive patients with nephropathy [28]. The decrease in urinary kallikrein is parallel to the existence of diabetic nephropathy with arterial hypertension [36]. Although the basal plasma renin activity in patients with type-2 diabetes was not significantly different from controls, both the renin activity and kallikrein levels increased after furosemide in controls while in diabetics this response was severely blunted, indicating early derangement of the KKS and the RAS as renal hemodynamic mechanisms heralding the onset of nephropathy [37]. Moreover, the suppressed renal KKS is evident not only in the whole kidney but in each nephron which is still functioning [38].

Furthermore, prior study has revealed that the reduction of kinin metabolism by ACEI might be involved in the beneficial effects exerted by these compounds in diabetic kidney functions [39]. Although an earlier study questions 
$\alpha$-SMA
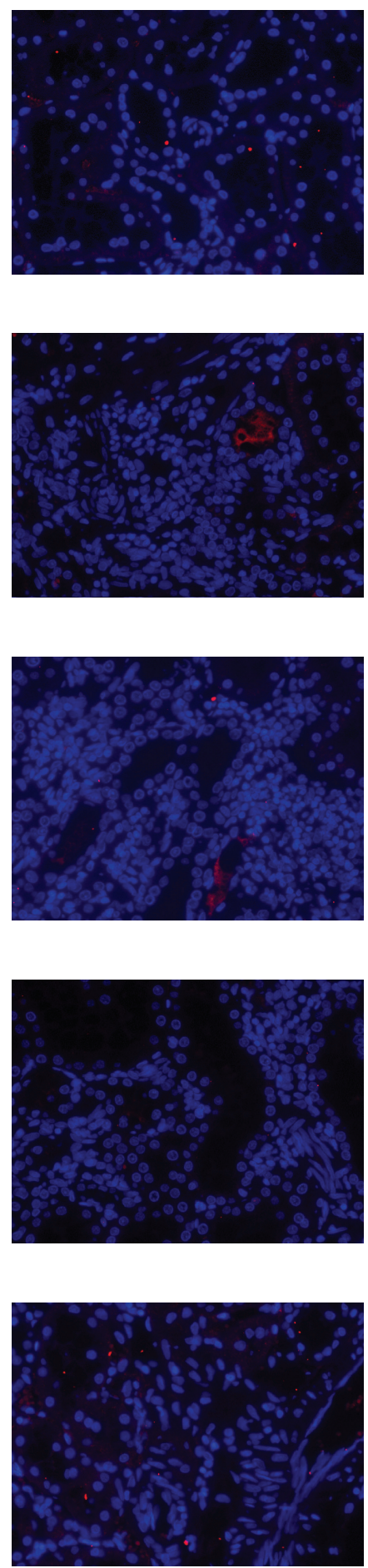

TGF- $\beta$

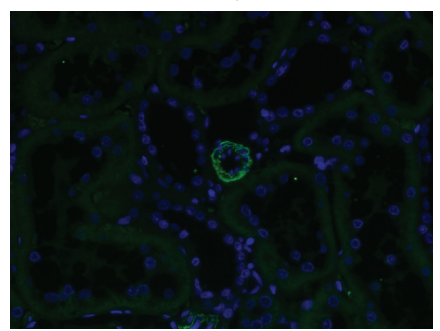

(a)

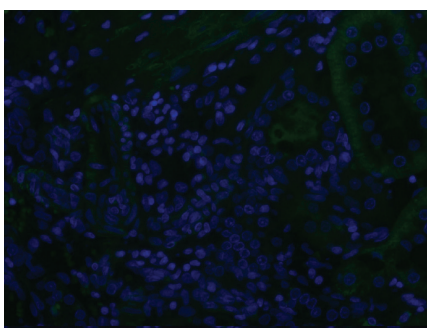

(b)

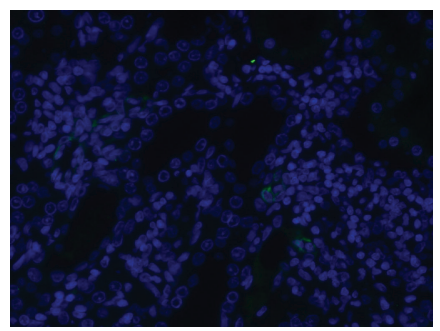

(c)

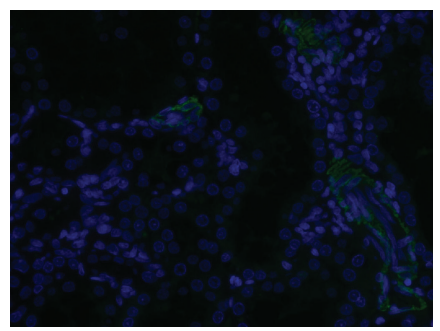

(d)
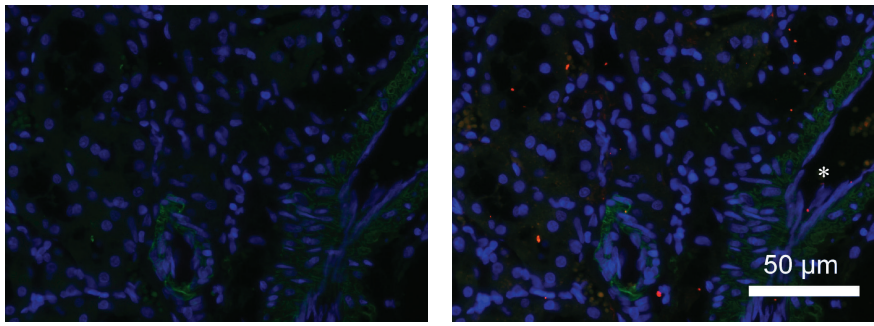

Merge
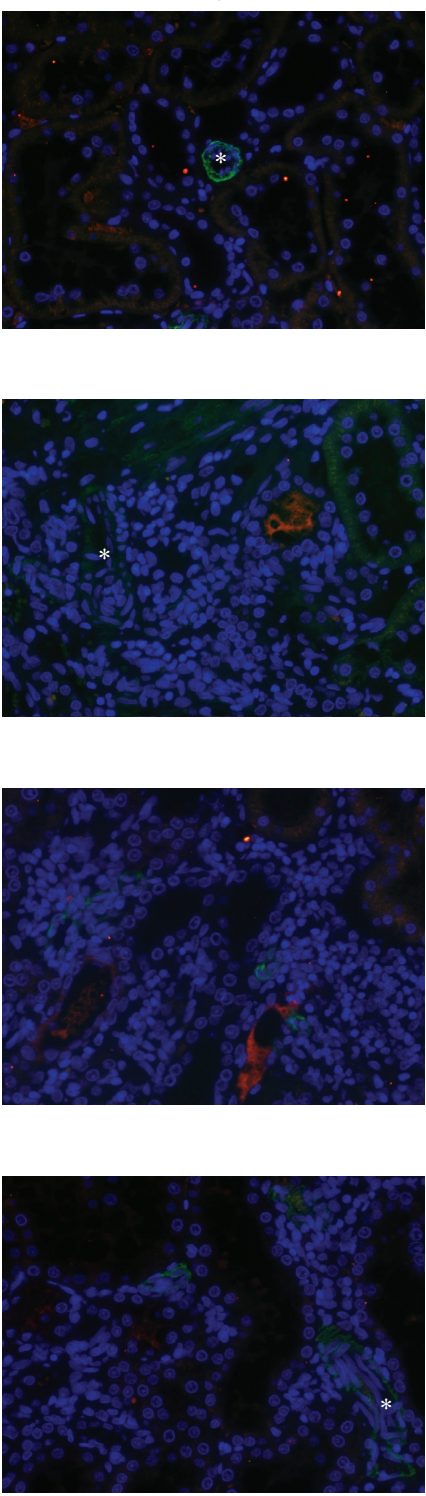

(e)

FIGURE 5: Immunofluorescence microscopy of $\alpha$-smooth muscle action and transforming growth factor- $\beta$. Kidney tissue sections (4 $\mu$ m) were stained with the activated fibroblast marker $\alpha$-smooth muscle action (green) and the profibrotic cytokine transforming growth factor- $\beta$ (red) and examined under a Zeiss A2 imaging microscope (Carl Zeiss, Hamburg, Germany). Both interstitial fibroblasts and vascular smooth muscle cells are positive for $\alpha$-smooth muscle action (green). Consistent with the immunostain of the phagocytic marker CD68 (Figure 4), inflammatory cells immunoreactive for transforming growth factor- $\beta$ (red) are predominantly interstitial large macrophages and frequently intermixed with infiltrating lymphocytes. (a) Normal control (CN); (b) model of diabetic nephropathy that never had any treatment (DN); (c) treatment with benazepril $10 \mathrm{mg} / \mathrm{kg} / \mathrm{d}$ (Ben); (d) treatment with irbesartan $30 \mathrm{mg} / \mathrm{kg} / \mathrm{d}$ (Irb); (e) combined treatment with benazepril $10 \mathrm{mg} / \mathrm{kg} / \mathrm{d}$ and irbesartan $30 \mathrm{mg} / \mathrm{kg} / \mathrm{d}$ (Ben and Irb). Original magnification, $\times 200$; scale bar, $50 \mu \mathrm{m}$. 
the postulate of a role of the KKS in the initiation of essential hypertension [40], interaction between the KKS and RAS may partially contribute to the multifactorial nature of diabetic nephropathy.

In addition to kidney haemodynamics modulated by the KKS-RAS counterbalance, inflammatory response appears to change after the initiation of diabetic nephropathy. The present study suggests potential correlation between the kallikrein activation and inflammatory amelioration. A recent experimental study showed the tissue kallikrein mediated proinflammatory pathways and activation of proteaseactivated receptor- 4 in proximal tubular epithelial cells [41]. Our study has several limitations. The kallikrein 1 level was identified by immunohistochemistry with light microscopy. The immunoreactivity of kallikrein 1 was confined in the kidney tubular epithelial cells. Levels of kallikrein 1 protein were not measured by Western blot. Due to lack of blood and urine samples, we could not measure the levels of plasma kallikrein 1, urinary kallikrein excretion, and kallikrein excretion rate ( $24 \mathrm{~h}$ excretion of urinary kallikrein/24 h creatinine clearance). Our future work will explore interactions between the kallikrein activation and inflammatory pathway by detailed measurements of kallikrein and cytokine levels in samples of plasma, kidney tissues, and urine.

Understanding of the RAS has shifted from the classical limited-proteolysis linear cascade to a cascade with multiple mediators, multiple receptors, and multifunctional enzymes. The homologue of ACE, ACE2, is insensitive to ACE inhibitors and forms angiotensin (1-7) [Ang-(1-7)] from angiotensin II (Ang II) with high efficiency. One important receptor for Ang-(1-7) is the Mas. The ACE2/Ang-(1-7)/Mas axis has putative role as an ACE-Ang II-AT1 receptor counterregulatory axis within the RAS $[42,43]$. In the present study, the dual RAS blockade exerted significant blood glucose lowering action for renoprotection. A landmark study has shown that the combination of losartan and lisinopril was associated with reduced Ang II formation and elevated cardiac ACE2 activity for increased Ang II metabolism [44]. Moreover, the renal cortex ACE2 formation was upregulated with increased levels of Ang-(1-7) in the urine of animals after ACEI or ARB [45]. These findings provide mechanistic insight of this renoprotective axis of the RAS. Indeed, the ACE2/Ang(1-7)/Mas axis can inhibit hepatic insulin resistance [46]. Furthermore, Ang-(1-7) generated by proximal tubular ACE2 inhibits Ang II-stimulated MAPK phosphorylation and TGF$\beta$ expression in proximal tubular cells [47]. Taken together, the ACE2/Ang-(1-7)/Mas axis exerts protective actions in diabetes, chronic kidney disease, hypertension, and other cardiovascular and metabolic disorders [48].

In the present study, we have no intension to promote the combined treatment of dual RAS blockade due to the multifactorial nature of diabetic nephropathy. Furthermore, a new clinical trial also provides evidence that combination therapy with ACEI and ARB is associated with an increased risk of adverse events among patients with diabetic nephropathy [49]. Taken together, new research questions remain for the role of kallikrein as a therapeutic target in diabetic nephropathy $[18,35,50,51]$.
In conclusion, kidney tissue kallikrein activation and inflammatory amelioration may underlie the renoprotective effects by the RAS blockade in diet-induced diabetic nephropathy. Further investigations are required to dissect the mechanistic interactions between the kallikrein-kinin system and renin-angiotensin system.

\section{Conflict of Interests}

The authors have declared that no conflict of interests exists.

\section{Authors' Contribution}

Xia Zou and Xiao-xi Zhang contributed equally to this study.

\section{Acknowledgments}

This work was supported by grants from the Guilin Medical University (KY2011002), Program for Innovative Research Team of Guilin Medical University (PIRTGMU), and National Natural Science Foundation of China (81270934).

\section{References}

[1] P. J. Held, F. K. Port, R. L. Webb et al., "The United States Renal Data System's 1991 annual data report: an introduction," American Journal of Kidney Diseases, vol. 18, no. 5, supplement 2, pp. 1-16, 1991.

[2] W. Yang, J. Lu, J. Weng et al., "Prevalence of diabetes among men and women in China," The New England Journal of Medicine, vol. 362, no. 12, pp. 1090-1101, 2010.

[3] Y. Xu, L. Wang, J. He et al., "Prevalence and control of diabetes in Chinese adults," The Journal of the American Medical Association, vol. 310, no. 9, pp. 948-959, 2013.

[4] L. Zhang, F. Wang, L. Wang et al., "Prevalence of chronic kidney disease in China: a cross-sectional survey," The Lancet, vol. 379, no. 9818, pp. 815-822, 2012.

[5] C. Y. Pan, L. T. Ho, S. Soegondo et al., "Prevalence of albuminuria and cardiovascular risk profile in a referred cohort of patients with type 2 diabetes: an Asian perspective," Diabetes Technology and Therapeutics, vol. 10, no. 5, pp. 397-403, 2008.

[6] E. Ritz, I. Rychlik, F. Locatelli, and S. Halimi, "End-stage renal failure in type 2 diabetes: a medical catastrophe of worldwide dimensions," American Journal of Kidney Diseases, vol. 34, no. 5, pp. 795-808, 1999.

[7] R. E. Schmieder, G. Bakris, and M. R. Weir, "Telmisartan in incipient and overt diabetic renal disease," Journal of Nephrology, vol. 24, no. 3, pp. 263-273, 2011.

[8] S. Anderson and R. Komers, "Inhibition of the reninangiotensin system: is more better?" Kidney International, vol. 75, no. 1, pp. 12-14, 2009.

[9] J. L. Finch, E. B. Suarez, K. Husain et al., "Effect of combining an ACE inhibitor and a VDR activator on glomerulosclerosis, proteinuria, and renal oxidative stress in uremic rats," American Journal of Physiology -Renal Physiology, vol. 302, no. 1, pp. 141$149,2012$.

[10] J. Allard, M. Buléon, E. Cellier et al., "ACE inhibitor reduces growth factor receptor expression and signaling but also albuminuria through B2-kinin glomerular receptor activation 
in diabetic rats," The American Journal of Physiology: Renal Physiology, vol. 293, no. 4, pp. F1083-F1092, 2007.

[11] B. M. Brenner, M. E. Cooper, D. de Zeeuw et al., "Effects of losartan on renal and cardiovascular outcomes in patients with type 2 diabetes and nephropathy," The New England Journal of Medicine, vol. 345, no. 12, pp. 861-869, 2001.

[12] H.-H. Parving, H. Lehnert, J. Brochner-Mortensen, R. Gomis, S. Andersen, and P. Arner, "The effect of irbesartan on the development of diabetic nephropathy in patients with type 2 diabetes," The New England Journal of Medicine, vol. 345, no. 12, pp. 870-878, 2001.

[13] M. Ruiz-Ortega, O. Lorenzo, M. Rupérez, V. Esteban, S. Mezzano, and J. Egido, "Renin-angiotensin system and renal damage: emerging data on angiotensin II as a proinflammatory mediator," Contributions to Nephrology, vol. 29, no. 135, pp. 123137, 2001.

[14] G. T. McInnes, K. P. J. O’Kane, H. Istad, S. KeinänenKiukaanniemi, and H. F. C. M. van Mierlo, "Comparison of the AT1-receptor blocker, candesartan cilexetil, and the ACE inhibitor, lisinopril, in fixed combination with low dose hydrochlorothiazide in hypertensive patients," Journal of Human Hypertension, vol. 14, no. 4, pp. 263-269, 2000.

[15] S. M. Nauli, "An ACE inhibitor improves vascular outcomes in a PKD model," The American Journal of Physiology-Renal Physiology, vol. 301, no. 5, p. F958, 2011.

[16] L. Tylicki, M. Renke, P. Rutkowski et al., "Dual blockade of the renin-angiotensin-aldosterone system with high-dose angiotensin-converting enzyme inhibitor for nephroprotection: an open, controlled, randomized study," Scandinavian Journal of Urology and Nephrology, vol. 42, no. 4, pp. 381-388, 2008.

[17] T. Shiraishi, Y. Tamura, K. Taniguchi et al., "Combination of ACE inhibitor with nicorandil provides further protection in chronic kidney disease," The American Journal of PhysiologyRenal Physiology, vol. 307, no. 12, pp. 1313-1322, 2014.

[18] M. Kakoki and O. Smithies, "The kallikrein-kinin system in health and in diseases of the kidney," Kidney International, vol. 75, no. 10, pp. 1019-1030, 2009.

[19] G. Spinetti, O. Fortunato, D. Cordella et al., "Tissue Kallikrein is essential for invasive capacity of circulating proangiogenic cells," Circulation Research, vol. 108, no. 3, pp. 284-293, 2011.

[20] U. Nöthlings, H. Boeing, G. Maskarinec et al., "Food intake of individuals with and without diabetes across different countries and ethnic groups," European Journal of Clinical Nutrition, vol. 65, no. 5, pp. 635-641, 2011.

[21] E. Ritz and S. R. Orth, "Nephropathy in patients with type 2 diabetes mellitus," The New England Journal of Medicine, vol. 341, no. 15, pp. 1127-1133, 1999.

[22] H.-L. Zhao, Y. Sui, J. Guan et al., "Fat redistribution and adipocyte transformation in uninephrectomized rats," Kidney International, vol. 74, no. 4, pp. 467-477, 2008.

[23] H.-L. Zhao, P. C. Y. Tong, F. M. M. Lai, B. Tomlinson, and J. C. N. Chan, "Association of glomerulopathy with the 5'end polymorphism of the aldose reductase gene and renal insufficiency in type 2 diabetic patients," Diabetes, vol. 53, no. 11, pp. 2984-2991, 2004.

[24] H.-L. Zhao, F. M. M. Lai, P. C. Y. Tong, B. Tomlinson, and J. C. N. Chan, "Clinicopathologic characteristics of nodular glomerulosclerosis in Chinese patients with type 2 diabetes," American Journal of Kidney Diseases, vol. 44, no. 6, pp. 10391049, 2004.
[25] B. A. Perkins, L. P. Aiello, and A. S. Krolewski, "Diabetes complications and the renin-angiotensin system," The New England Journal of Medicine, vol. 361, no. 1, pp. 83-85, 2009.

[26] S. C. W. Tang, J. C. K. Leung, and K. N. Lai, “The kallikreinkinin system," Contributions to Nephrology, vol. 170, no. 3, pp. 145-155, 2011.

[27] A. Manto, P. Cotroneo, G. Porcelli et al., "Urinary kallikrein excretion in type 1 (insulin-dependent) diabetes mellitus," Diabetologia, vol. 36, no. 5, pp. 423-427, 1993.

[28] T. Baba, S. Murabayashi, T. Ishizaki, Y. Idol, K. Aoyagi, and K. Takebe, "Renal kallikrein in diabetic patients with hypertension accompanied by nephropathy," Diabetologia, vol. 29, no. 3, pp. 162-167, 1986.

[29] S. Bodin, C. Chollet, N. Goncalves-Mendes et al., "Kallikrein protects against microalbuminuria in experimental type I diabetes," Kidney International, vol. 76, no. 4, pp. 395-403, 2009.

[30] J. N. Harvey, A. A. Jaffa, H. S. Margolius, and R. K. Mayfield, "Renal kallikrein and hemodynamic abnormalities of diabetic kidney," Diabetes, vol. 39, no. 3, pp. 299-304, 1990.

[31] C. Tschöpe, A. Reinecke, U. Seidl et al., "Functional, biochemical, and molecular investigations of renal kallikrein-kinin system in diabetic rats," The American Journal of PhysiologyHeart and Circulatory Physiology, vol. 277, no. 6, pp. H2333H2340, 1999.

[32] S.-J. Kwak, J. Paeng, D. H. Kim et al., "Local kallikreinkinin system is involved in podocyte apoptosis under diabetic conditions," Apoptosis, vol. 16, no. 5, pp. 478-490, 2011.

[33] C. Zhao, P. Wang, X. Xiao et al., "Gene therapy with human tissue kallikrein reduces hypertension and hyperinsulinemia in fructose-induced hypertensive rats," Hypertension, vol. 42, no. 5, pp. 1026-1033, 2003.

[34] G. Yuan, J. Deng, T. Wang et al., “Tissue kallikrein reverses insulin resistance and attenuates nephropathy in diabetic rats by activation of phosphatidylinositol 3-kinase/protein kinase $\mathrm{B}$ and adenosine $5^{\prime}$-monophosphate-activated protein kinase signaling pathways," Endocrinology, vol. 148, no. 5, pp. 20162026, 2007.

[35] A. Riad, J. L. Zhuo, H. P. Schultheiss, and C. Tschöpe, “The role of the renal kallikrein-kinin system in diabetic nephropathy," Current Opinion in Nephrology and Hypertension, vol. 16, no. 1, pp. 22-26, 2007.

[36] F. J. P. Blanco, M. J. C. López, C. F. García, R. S. Sánchez, and A. R. Cuartero, "Renal kallikrein in diabetic nephropathy," Revista Clínica Española, vol. 188, no. 3, pp. 123-126, 1991.

[37] S. B. Lall, S. Kunchaparty, H. H. Siddiqui, and J. S. Bajaj, "Plasma renin activity and urinary kallikrein excretion in response to intravenous furosemide in diabetic patients," Acta Diabetologica Latina, vol. 27, no. 4, pp. 337-342, 1990.

[38] T. Ando, K. Shimamoto, N. Ura et al., "The renal KallikreinKinin system in renoparenchymal hypertension," Advances in Experimental Medicine and Biology, vol. 247, no. 21, pp. 127-132, 1989.

[39] J. Damas, N. Garbacki, and P. J. Lefebvre, "The kallikrein-kinin system, angiotensin converting enzyme inhibitors and insulin sensitivity," Diabetes/Metabolism Research and Reviews, vol. 20, no. 4, pp. 288-297, 2004.

[40] O. B. Holland, J. M. Chud, and H. Braunstein, "Urinary kallikrein excretion in essential and mineralocorticoid hypertension," Journal of Clinical Investigation, vol. 65, no. 2, pp. 347356, 1980. 
[41] W. H. Yiu, D. W. L. Wong, L. Y. Y. Chan, and et al, "Tissue kallikrein mediates pro-inflammatory pathways and activation of protease-activated receptor-4 in proximal tubular epithelial cells," PLoS ONE, vol. 9, no. 2, Article ID e88894, 2014.

[42] R. A. S. Santos, A. J. Ferreira, and A. C. Simões E Silva, "Recent advances in the angiotensin-converting enzyme 2angiotensin(1-7)-Mas axis," Experimental Physiology, vol. 93, no. 5, pp. 519-527, 2008.

[43] C. M. Ferrario and J. Varagic, "The ANG-(1-7)/ACE2/mas axis in the regulation of nephron function," American Journal of Physiology-Renal Physiology, vol. 298, no. 6, pp. F1297-F1305, 2010.

[44] C. M. Ferrario, J. Jessup, M. C. Chappell et al., "Effect of angiotensin-converting enzyme inhibition and angiotensin II receptor blockers on cardiac angiotensin-converting enzyme 2," Circulation, vol. 111, no. 20, pp. 2605-2610, 2005.

[45] C. M. Ferrario, J. Jessup, P. E. Gallagher et al., "Effects of renin-angiotensin system blockade on renal angiotensin-(1-7) forming enzymes and receptors," Kidney International, vol. 68, no. 5, pp. 2189-2196, 2005.

[46] X. Cao, F.-Y. Yang, Z. Xin, R.-R. Xie, and J.-K. Yang, “The ACE2/Ang-(1-7)/Mas axis can inhibit hepatic insulin resistance," Molecular and Cellular Endocrinology, vol. 393, no. 1-2, pp. 30-38, 2014.

[47] Z. Su, J. Zimpelmann, and K. D. Burns, "Angiotensin-(1-7) inhibits angiotensin II-stimulated phosphorylation of MAP kinases in proximal tubular cells," Kidney International, vol. 69, no. 12, pp. 2212-2218, 2006.

[48] M. Iwai and M. Horiuchi, "Devil and angel in the reninangiotensin system: ACE-angiotensin II-AT1 receptor axis vs. ACE2-angiotensin-(1-7)-Mas receptor axis," Hypertension Research, vol. 32, no. 7, pp. 533-536, 2009.

[49] L. F. Fried, N. Emanuele, J. H. Zhang et al., "Combined angiotensin inhibition for the treatment of diabetic nephropathy," The New England Journal of Medicine, vol. 369, no. 20, pp. 1892-1903, 2013.

[50] J. A. Phipps and E. P. Feener, "The kallikrein-kinin system in diabetic retinopathy: lessons for the kidney," Kidney International, vol. 73, no. 10, pp. 1114-1119, 2008.

[51] D. de Zeeuw, "The end of dual therapy with renin-angiotensinaldosterone system blockade?" The New England Journal of Medicine, vol. 369, no. 20, pp. 1960-1962, 2013. 


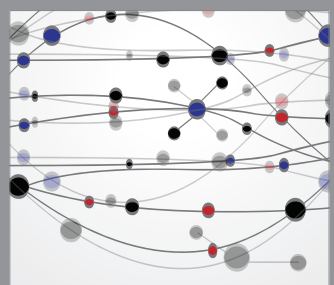

The Scientific World Journal
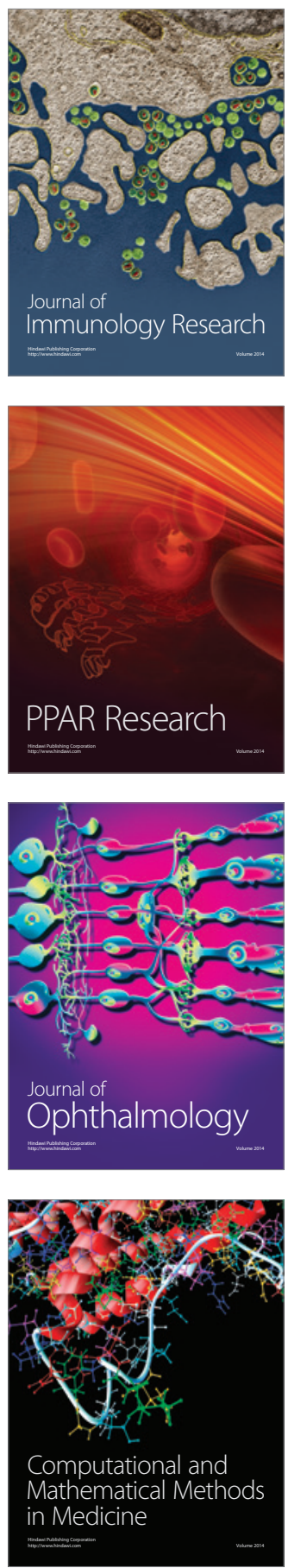

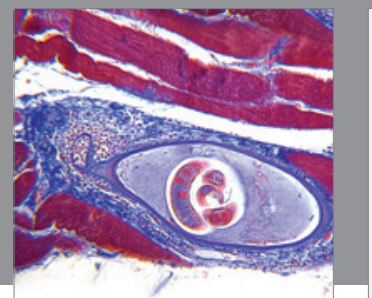

Gastroenterology

Research and Practice
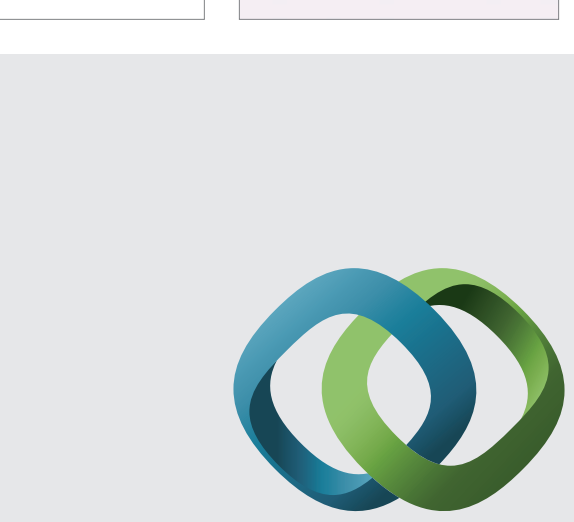

\section{Hindawi}

Submit your manuscripts at

http://www.hindawi.com
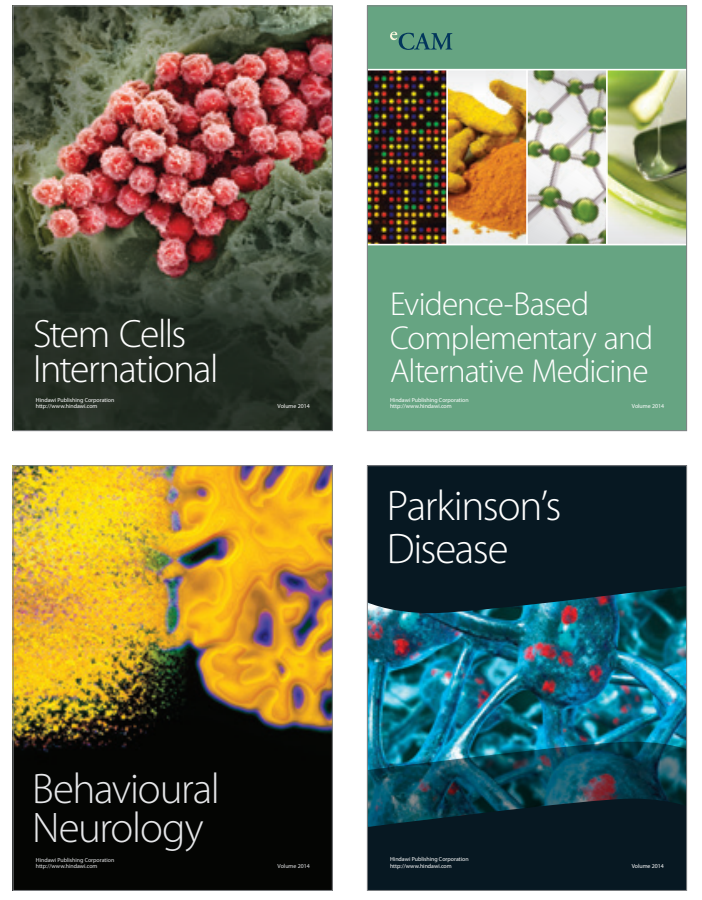
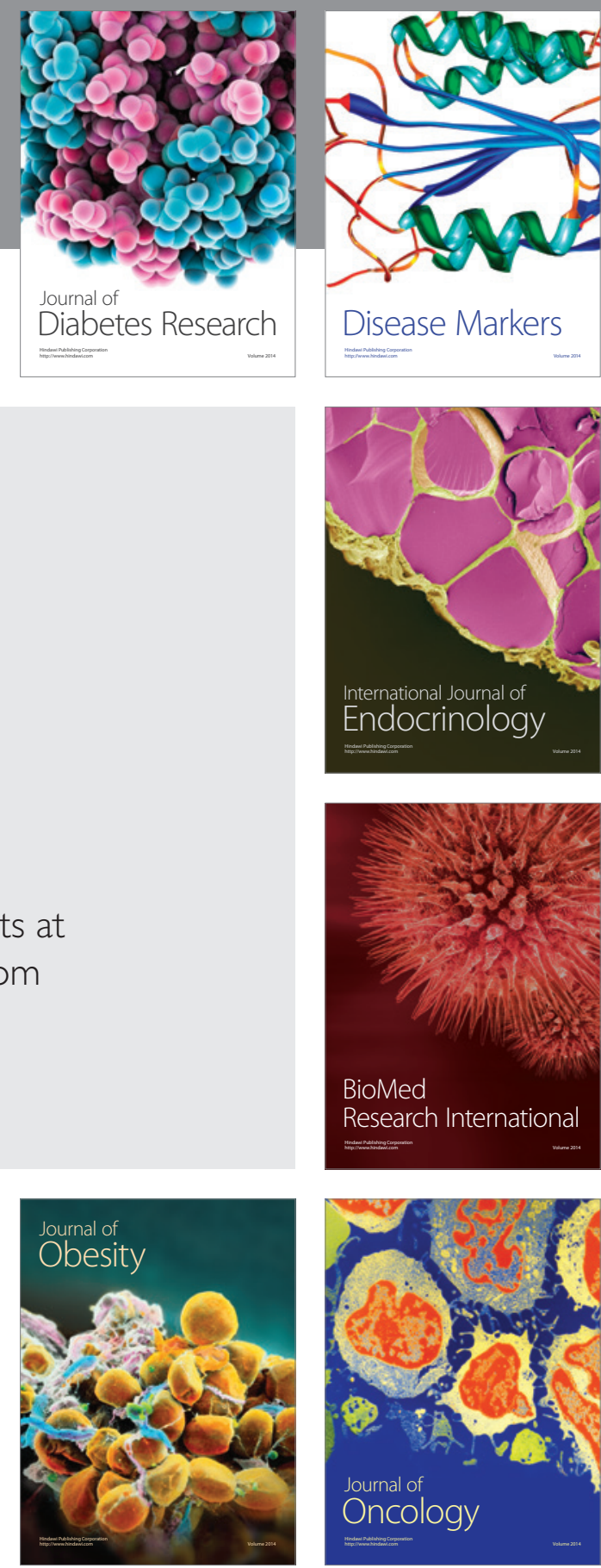

Disease Markers
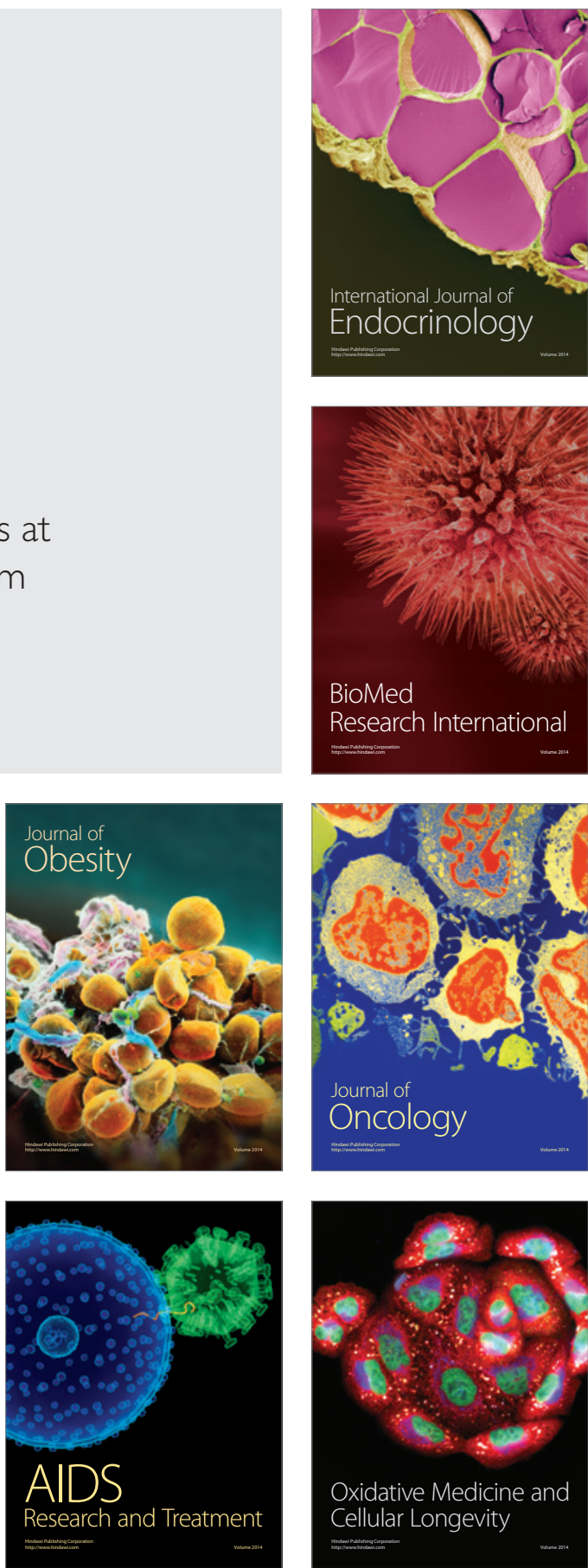